\title{
Advanced Stage Hepatocellular Carcinoma Successfully Treated with Transarterial Radioembolization and Multi-tyrosine Kinase Inhibitor Therapy
}

Myung Ji Goh, Wonseok Kang, Dong Hyun Sinn, Geum-Youn Gwak, Yong-Han Paik, Moon Seok Choi, Joon Hyeok Lee, Kwang Cheol Koh, Seung Woon Paik

Department of Internal Medicine, Samsung Medical Center, Sungkyunkwan University School of Medicine, Seoul, Korea

Received Dec. 31, 2019

Revised Feb. 1, 2020

Accepted Mar. 1, 2020
Transarterial radioembolization (TARE) with yttrium-90 microspheres has become widely utilized in managing hepatocellular carcinoma (HCC). The utility of TARE is expanding with new insights through experiences from real-world practice and clinical trials, and recently published data suggest that TARE in combination with sorafenib may improve the overall survival in selected patients. Here, we report a case of advanced stage HCC that was successfully treated with TARE and sorafenib. The patient achieved complete response (CR) at 12 months after the initial treatment with TARE and sorafenib, followed by additional transarterial chemoembolization and proton beam therapy for local tumor recurrence at 19-month post-TARE. The patient was followed up every 3 months thereafter and still achieved CR both biochemically and radiologically for the following 12 months. A combination strategy of TARE and systemic therapy may be a useful alternative treatment option for selected patients with advanced stage HCC. (J Liver Cancer 2020;20:160-166)

Keywords: Hepatocellular carcinoma; Yttrium-90; Radioembolization; Sorafenib; Tyrosine kinase inhibitor

\section{INTRODUCTION}

Hepatocellular carcinoma (HCC) is the most common primary malignancy of the liver and the second leading cause of cancer-related mortality worldwide. ${ }^{1,2}$ Based on the Barcelona Clinic Liver Cancer (BCLC) staging system, stage 0 or A patients with very early- or early-stage disease are eligible for curative therapies including ablative therapies, surgical resec-

\section{Corresponding author : Wonseok Kang}

Department of Internal Medicine, Samsung Medical Center, Sungkyunkwan University School of Medicine, 81 Irwon-ro, Gangnam-gu, Seoul 06351, Korea

Tel. +82-2-3410-3409, Fax. +82-2-3410-6983

E-mail;wonseok1202.kang@samsung.com

https://orcid.org/0000-0001-9578-8424 tion, or liver transplant. ${ }^{3}$ However, significantly few patients are eligible for these curative options due to the presence of advanced disease upon presentation. For intermediate or advanced stage HCCs, standard of care includes transarterial chemoembolization (TACE) or systemic therapy. ${ }^{3}$ Recently, transarterial radioembolization (TARE) with yttrium-90 $\left.{ }^{90} \mathrm{Y}\right)$ microspheres has become an increasingly popular treatment option. ${ }^{4}$ TARE is performed by image-guided deposition of radiated ${ }^{90} \mathrm{Y}$ microspheres to the tumor through the hepatic artery, which is the most common source of perfusion for HCC. TARE with ${ }^{90} \mathrm{Y}$ microsphere has emerged as an alternative treatment to TACE, and this treatment option has comparable survival rate with TACE but results in superior quality of life compared to TACE. Moreover, this treat-

Copyright $\odot 2020$ by The Korean Liver Cancer Association. All rights reserved.

This is an Open Access article distributed under the terms of the Creative Commons Attribution Non-Commercial License (http://creativecommons.org/licenses/by-nc/4.0/) which permits unrestricted non-commercial use, distribution, and reproduction in any medium, provided the original work is properly cited. 
ment is applicable for advanced stage HCC with reduced risk of ischemic hepatitis considering that arterial occlusion is not commonly observed in this treatment. ${ }^{5-7}$ For patients with advanced HCC, sorafenib is the first-line therapeutic option; however, sorafenib has a more modest survival benefit compared to placebos. ${ }^{8-10}$ Some in vitro and in vivo studies had investigated the possible synergistic effect between TARE and sorafenib therapy. ${ }^{11,12}$ Moreover, according to a palliative sub-study of the Sorafenib and Micro-therapy Guided by Primovist Enhanced magnetic resonance imaging (MRI) in Patients With Inoperable Liver Cancer (SORAMIC trial, NCT01126645), the interim safety analysis reassures that ${ }^{90} \mathrm{Y}$ radioembolization as a sequential approach followed by an escalation scheme for sorafenib does not lead to increased toxicity. ${ }^{13}$ Herein, we describe the case of an advanced stage HCC patient who showed a favorable response following a
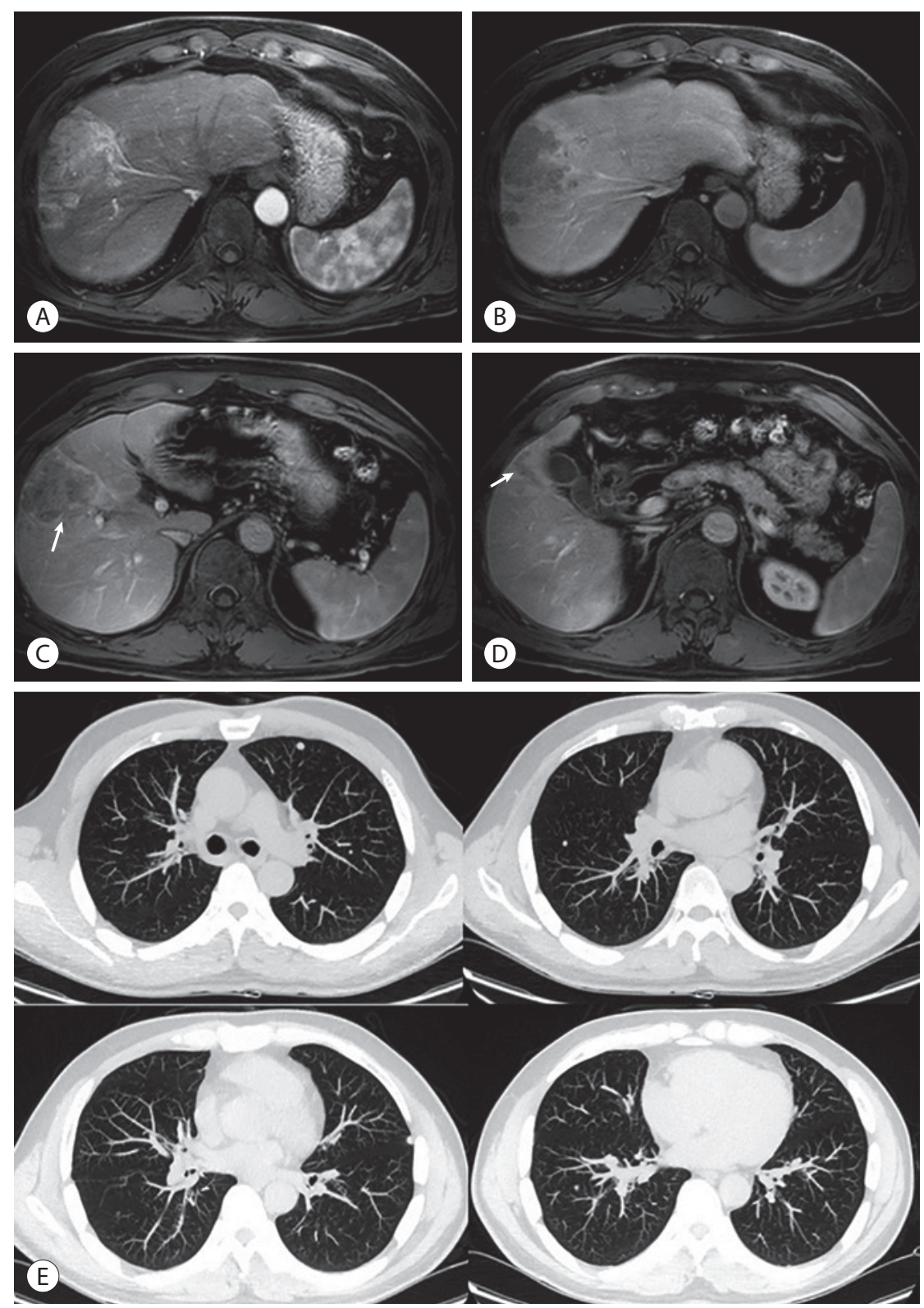

Figure 1. Initial liver dynamic magnetic resonance image findings. Multinodular mass showed enhancement on the arterial phase (A) and washout on the portal phase (B-D). The tumor thrombosis involved in the portal vein branches and mid-hepatic vein (arrows). (E) Initial chest computed tomography findings. Multiple pulmonary metastases in both lungs were observed. 
combination treatment with TARE and sorafenib.

\section{CASE REPORT}

\section{Clinical findings}

A 56-year-old male patient presented with multinodular liver masses incidentally detected on screening ultrasonography at another hospital. The patient was diagnosed with chronic hepatitis B 20 years prior, but he was not under regular HCC surveillance. The patient had consumed 1 bottle of soju (Korean distilled liquor with an alcohol content of 1520\%), 3 times a week, but abstained from alcohol since he was diagnosed with HCC. He had no associated symptoms,
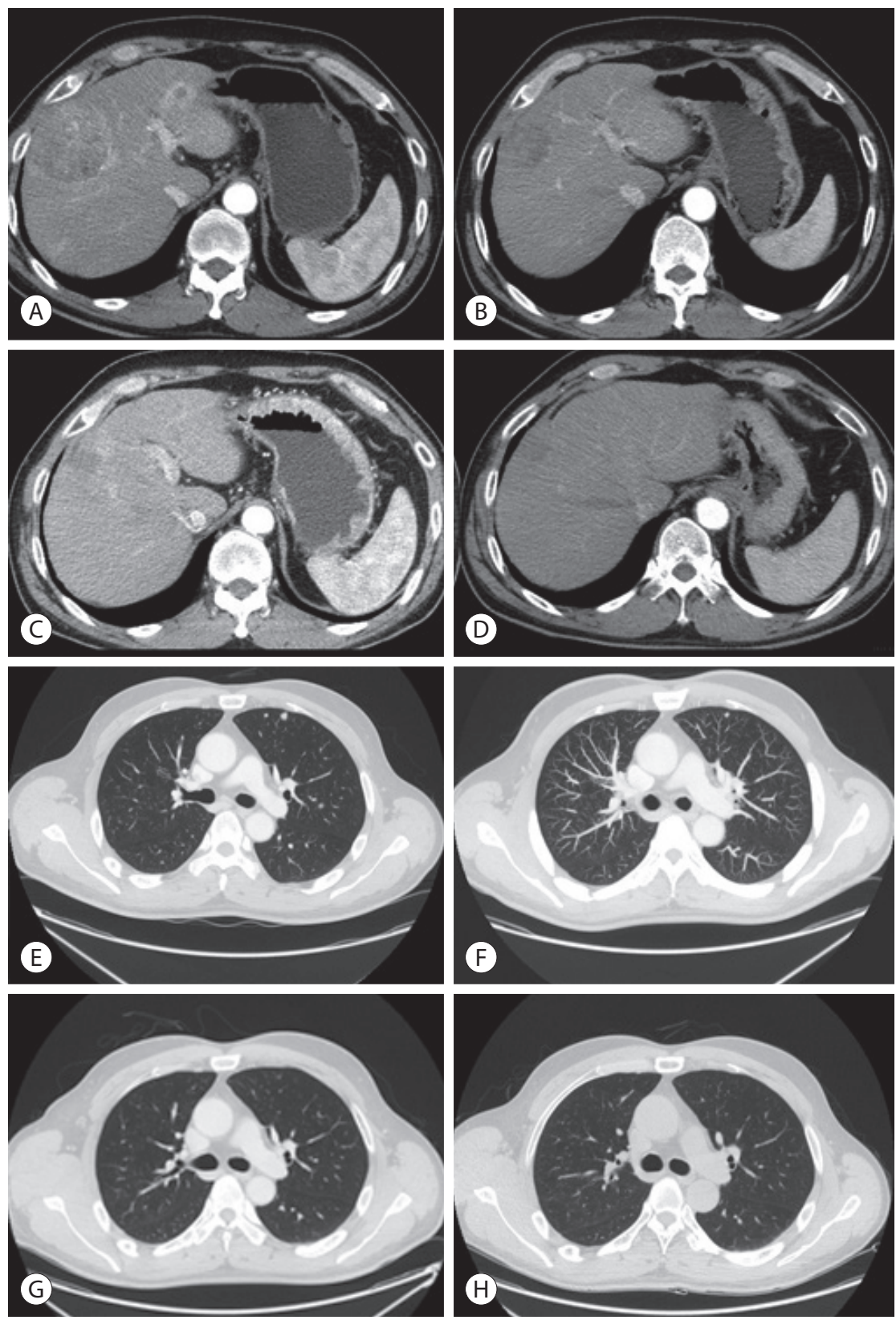

Figure 2. Follow-up liver dynamic computed tomography (CT) findings at 1-month (A), 3-month (B), 6-month (C), and 12-month (D) post-transarterial radioembolization (TARE). Follow-up chest CT findings at 1-month (E), 3-month (F), 6-month (G), and 12-month (H) post-TARE. 
and there were no remarkable findings on physical examination. Initial laboratory findings were as follows: white blood cell, 4,200/ $\mu \mathrm{L}$; hemoglobin, $15.0 \mathrm{~g} / \mathrm{dL}$; platelet, 137,000/ $\mu \mathrm{L}$; total bilirubin, $0.8 \mathrm{mg} / \mathrm{dL}$; albumin, $4.1 \mathrm{~g} / \mathrm{dL}$; international normalized ratio, 1.08; aspartate aminotransferase, 92 IU/L; alanine aminotransferase, $51 \mathrm{IU} / \mathrm{L}$; and serum hepatitis B virus DNA 7,787,219 IU/mL. Alpha-fetoprotein (AFP) level was 33,996 ng/mL, and protein induced by vitamin $\mathrm{K}$ absence or antagonist-II (PIVKA-II) level was $51 \mathrm{mAU} / \mathrm{mL}$. The liver function was preserved (Child-Pugh score, 5).

\section{Image findings}

Initial contrast-enhanced multiphasic liver computed to- mography (CT) revealed a 6-cm-sized liver mass in segment (S) 5/8 with multiple intrahepatic satellite nodules and enlarged lymph nodes in the common hepatic artery area and portacaval space. The liver mass showed typical radiological features of HCC-hyperenhancement on the arterial phase and washout on portal venous and delayed phases. Liver dynamic MRI revealed multifocal tumor thrombosis involving S5/S8 portal vein branches and middle hepatic vein (Fig. 1AD). Chest CT revealed multiple pulmonary metastases in both lungs (Fig. 1E).

\section{Diagnosis and treatment}

The patient was diagnosed with an advanced stage HCC
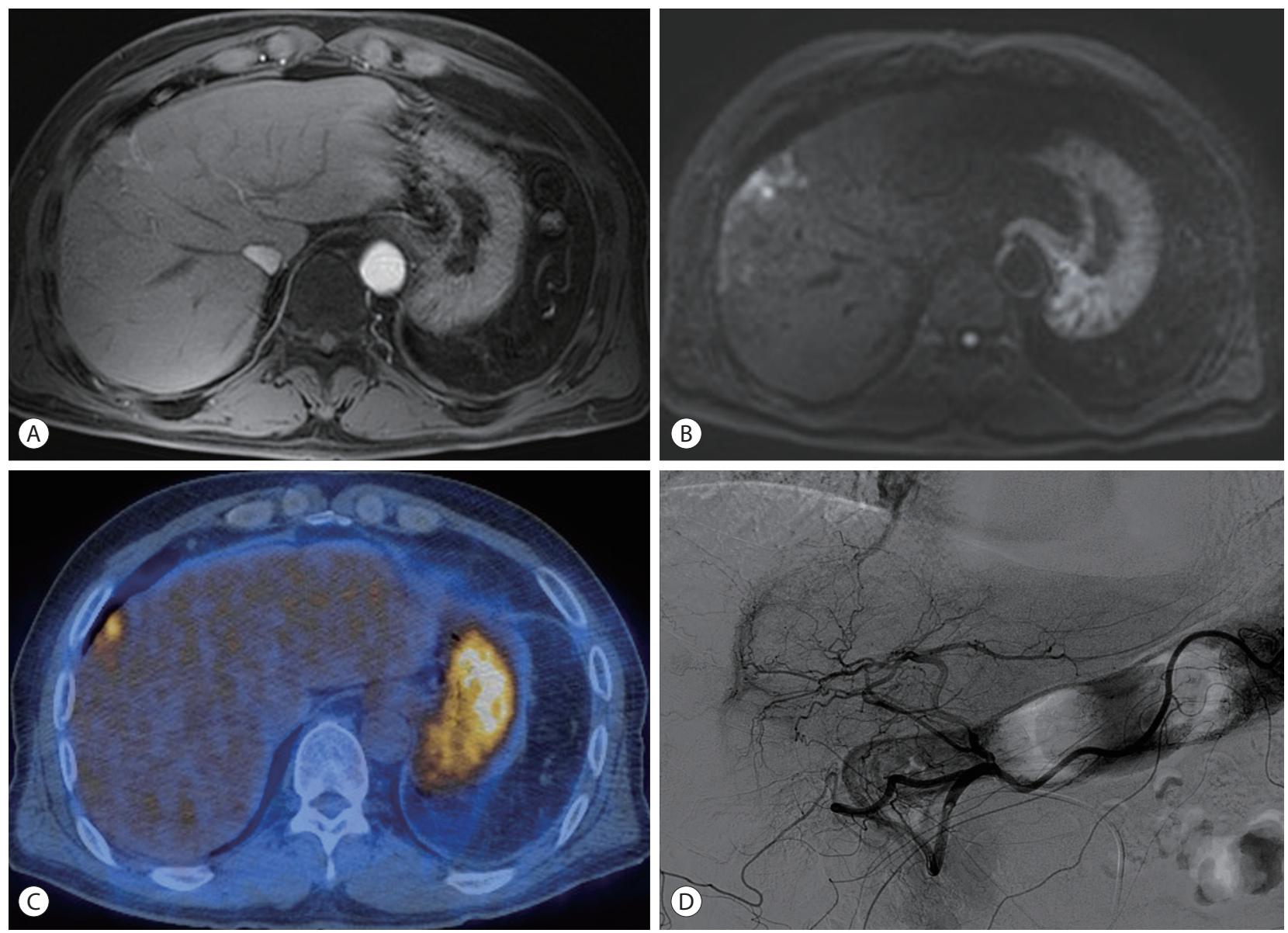

Figure 3. Follow-up liver dynamic magnetic resonance imaging at 19-month post- transarterial radioembolization showing a subcentimeter-sized enhancing viable tumor at the top of segment $8 / 4$ lesion on the arterial phase (A) with diffusion restriction (B). A focal hypermetabolic lesion (maximum standardized uptake value $\left[\mathrm{SUV}_{\max }\right]=5.3$ ) at the corresponding lesion in positron emission tomography-computed tomography (C). Hepatic angiography. Multiple punctate tumor stainings at segment $8 / 4$ were observed (D). 
(BCLC stage C, modified Union for International Cancer Control T4N1M1, stage IVB) without liver biopsy since typical features of HCC in imaging results were observed. ${ }^{14}$ The patient was treated with ${ }^{90} \mathrm{Y}$ TARE for the local control of intrahepatic lesions, followed by sorafenib for the systemic control of the tumor. For underlying chronic hepatitis B and cirrhosis, entecavir $0.5 \mathrm{mg}$ was administered. TARE was performed using TheraSphere ${ }^{\circledR}$ glass microspheres (BTG PLC, London, UK); the total infused radiation activity was 14 gigabecquerel (GBq) with $3 \mathrm{GBq}$ for $\mathrm{S} 4,3 \mathrm{GBq}$ for $\mathrm{S} 5$, and 8 GBq for S5/S8. The dose was calculated using the Medical Internal Radiation Dose method, as recommended by the manufacturer. No adverse events or clinical signs of hepatic decompensation were observed after the procedure. The patient started to take sorafenib $400 \mathrm{mg}$ twice daily 1-month
post-TARE. However, sorafenib dose was reduced to $400 \mathrm{mg}$ once daily since grade 2 adverse events with hand-foot-skin reaction (HFSR), which developed 2 weeks after beginning sorafenib, were observed.

At 3-month post-TARE, partial response was achieved according to the modified Response Evaluation Criteria In Solid Tumors (RECIST) criteria. ${ }^{15}$ Multifocal infiltrative lesions in the right hepatic lobe significantly decreased in extent with reduced arterial enhancement. Furthermore, metastatic lung nodules also decreased in number and size. AFP level decreased greater than 10 -fold at 1,290 ng/mL.

At 12-month post-TARE, complete response (CR) was achieved with the absence of arterial enhancement on target liver lesion, and metastatic nodules in both lungs (Fig. 2) and portal/hepatic vein thrombosis were already not observed.

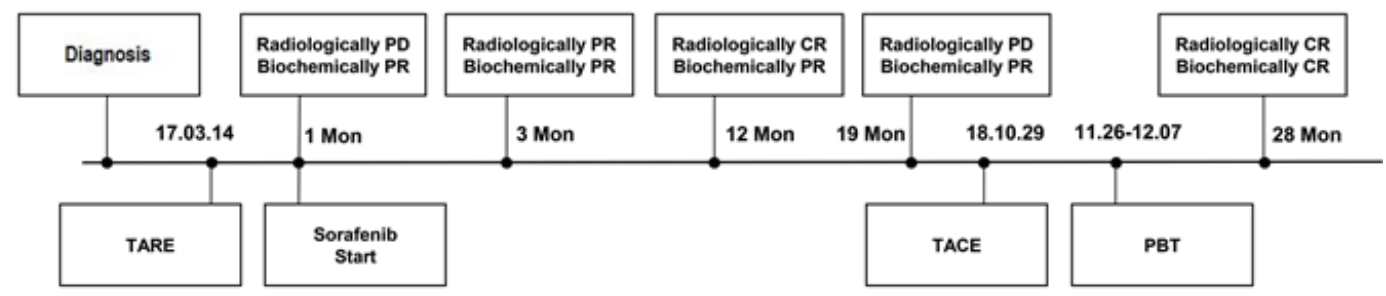

AFP

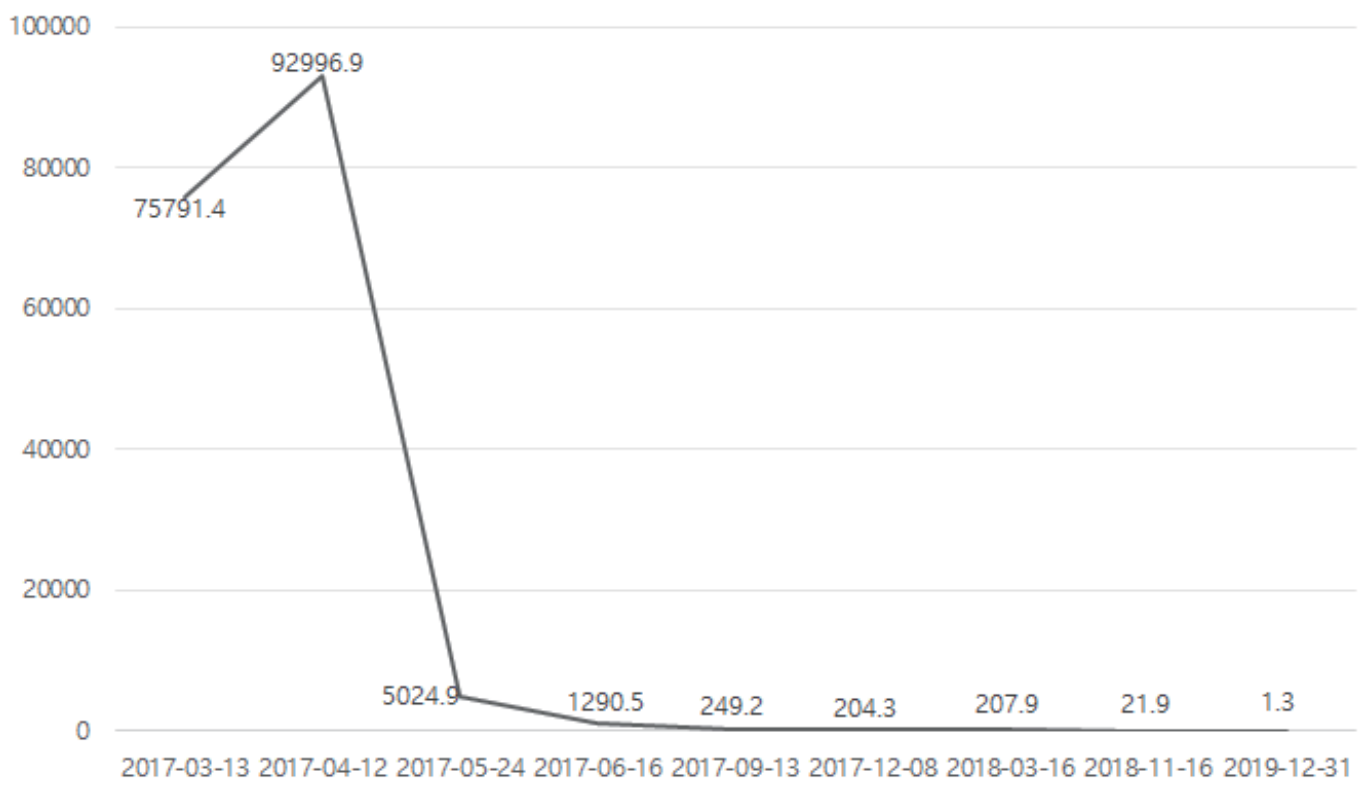

Figure 4. Clinical progress including treatment modalities, treatment response, and change in tumor markers (bottom) during the follow-up period. Mon, months; TARE, transarterial radioembolization; TACE, transarterial chemoembolization; PBT, proton beam therapy; PD, progressive disease; PR, partial response; $C R$, complete response; AFP, alpha-fetoprotein. 
Serum PIVKA-II level was within the normal range at $26 \mathrm{mAU} / \mathrm{mL}$; however, serum AFP level remained slightly elevated at $249 \mathrm{ng} / \mathrm{mL}$. After achieving radiological CR, the patient refused further sorafenib treatment considering that grade 2 adverse events with HFSR were observed during sorafenib treatment.

At 19-month post-TARE, an arterial enhancing nodule measuring up to $8 \mathrm{~mm}$ was observed in the subcapsular area of $\mathrm{S} 8 / \mathrm{S} 4$, which displayed diffusion restriction in liver dynamic MRI and an uptake of maximum standardized uptake value $\left(\mathrm{SUV}_{\max }\right) 5.3$ in positron emission tomography-CT, consistent with local tumor recurrence with suspicious tumor thrombosis at the portal vein of S4 (P4) (Fig. 3). Subsequently, additional TACE was performed for the viable tumor at $\mathrm{S} 8 / \mathrm{S} 4$ followed by a consolidation proton beam therapy of 6.6 cobalt gray equivalent on S4 and P4. AFP level was within the normal range 2 months after TACE and proton beam therapy. The patient was followed up every 3 months thereafter and still achieved CR both biochemically and radiologically for the following 12 months. Clinical course is described in Fig. 4 based on treatment modality, treatment response, and change in AFP.

\section{DISCUSSION}

Here, we present an interesting case of advanced stage HCC that was successfully treated with TARE and sorafenib. The patient achieved CR through initial treatment with TARE and sorafenib, followed by additional TACE and proton beam therapy for local tumor recurrence.

According to the current practice guidelines, systemic therapy with a multi-tyrosine kinase inhibitor (TKI) (i.e., sorafenib or lenvatinib) is recommended as the first-line therapeutic option for advanced stage HCC. ${ }^{3,16}$ However, only a few proportion of patients with advanced stage HCC benefit from TKIs, with modest survival gain of only 2-3 months. ${ }^{8-10}$ Some studies, including five large randomized trials, were conducted to evaluate the effectiveness of TACE and sorafenib combination therapy. These studies confirmed that the overall survival rate was lower in this combination therapy than in sorafenib therapy alone. ${ }^{17}$ TARE is better tol- erated than TACE, which may allow a higher dose and longer length of therapy with sorafenib. ${ }^{18}$ Additionally, some in vitro and in vivo studies found that sorafenib may be synergistic with TARE considering the antiangiogenic effect of sorafenib, which allowed the tumor vessels to deliver oxygen more efficiently to the core of HCC tumors, possibly enhancing the effect of radiation-induced tumor regression. ${ }^{11,12}$

On the contrary to theoretical rationale, the first large randomized controlled trial, SORAMIC, failed to demonstrate a higher survival benefit of TARE and sorafenib combination therapy than sorafenib therapy alone for the palliative treatment of unresectable HCC. However, a possible overall survival benefit was observed in some subgroups including younger patients aged $<65$ years, those with nonalcoholic cirrhosis, and those without cirrhosis. ${ }^{19}$ The current patient was a good candidate for ${ }^{90} \mathrm{Y}$ TARE plus sorafenib combination therapy considering his young age and preserved liver function.

Another phase 3 prospective study, the TheraSphere in the Treatment of Patients with Unresectable Hepatocellular Carcinoma (STOP-HCC trial, NCT01556490), that comprises a larger sample size that aimed to detect a difference in the overall survival between the combined therapy and sorafenib alone therapy in unresectable HCC is ongoing, and preliminary results of the two first interim analyses support the continuation of the study. ${ }^{20}$

Safety analyses in the SORAMIC study reported higher incidence of grade 3-4 adverse events in the combination therapy group than in the sorafenib only group. ${ }^{13}$ Grade 2 adverse events with HFSR were developed in this case, and the patient refused further sorafenib treatment after achieving radiological CR.

In conclusion, we described a case of advanced stage HCC that was successfully treated with TARE and sorafenib without severe adverse events. Thus, a combination strategy of TARE and systemic therapy may be a useful alternative treatment option for selected patients with advanced stage HCC.

\section{ACKNOWLEDGMENTS}

The present case report was approved by the Institutional 
Review Boards (IRBs) of the Samsung Medical Center (IRB No.2020-03-047). Written informed consent was obtained from the patient for publication of this case report. The authors acknowledge grant support of the National Research Foundation of Korea (NRF) (2016R1C1B2015463, W.K).

\section{Conflicts of Interest}

The authors have no conflicts of interest to disclose.

\section{REFERENCES}

1. Bray F, Ferlay J, Soerjomataram I, Siegel RL, Torre LA, Jemal A. Global cancer statistics 2018: GLOBOCAN estimates of incidence and mortality worldwide for 36 cancers in 185 countries. CA Cancer J Clin 2018;68:394-424.

2. Rawla P, Sunkara T, Muralidharan P, Raj JP. Update in global trends and aetiology of hepatocellular carcinoma. Contemp Oncol (Pozn) 2018;22:141-150.

3. Heimbach JK, Kulik LM, Finn RS, Sirlin CB, Abecassis MM, Roberts $L R$, et al. AASLD guidelines for the treatment of hepatocellular carcinoma. Hepatology 2018;67:358-380.

4. Salem R, Gabr A, Riaz A, Mora R, Ali R, Abecassis M, et al. Institutional decision to adopt $Y 90$ as primary treatment for hepatocellular carcinoma informed by a 1,000-patient 15-year experience. Hepatology 2018;68:1429-1440.

5. Kim DY, Han KH. Transarterial chemoembolization versus transarterial radioembolization in hepatocellular carcinoma: optimization of selecting treatment modality. Hepatol Int 2016;10:883-892.

6. Sangro B, Salem R. Transarterial chemoembolization and radioembolization. Semin Liver Dis 2014;34:435-443.

7. Moreno-Luna LE, Yang JD, Sanchez W, Paz-Fumagalli R, Harnois DM, Mettler TA, et al. Efficacy and safety of transarterial radioembolization versus chemoembolization in patients with hepatocellular carcinoma. Cardiovasc Intervent Radiol 2013;36:714-723.

8. Bruix J, Cheng AL, Meinhardt G, Nakajima K, De Sanctis Y, Llovet J. Prognostic factors and predictors of sorafenib benefit in patients with hepatocellular carcinoma: analysis of two phase III studies. J Hepatol 2017;67:999-1008.

9. Cheng AL, Kang YK, Chen Z, Tsao CJ, Qin S, Kim JS, et al. Efficacy and safety of sorafenib in patients in the Asia-Pacific region with advanced hepatocellular carcinoma: a phase III randomised, dou- ble-blind, placebo-controlled trial. Lancet Oncol 2009;10:25-34.

10. Llovet JM, Ricci S, Mazzaferro V, Hilgard P, Gane E, Blanc JF, et al. Sorafenib in advanced hepatocellular carcinoma. N Engl J Med 2008;359:378-390.

11. Plastaras JP, Kim SH, Liu YY, Dicker DT, Dorsey JF, McDonough J, et al. Cell cycle dependent and schedule-dependent antitumor effects of sorafenib combined with radiation. Cancer Res 2007;67:94439454.

12. Pracht M, Edeline J, Lepareur N, Lenoir L, Ardisson V, Clement B, et al. In vitro demonstration of synergy/additivity between (188) rhenium and sorafenib on hepatoma lines: preliminary results. Anticancer Res 2013;33:3871-3877.

13. Ricke J, Bulla K, Kolligs F, Peck-Radosavljevic M, Reimer P, Sangro $B$, et al. Safety and toxicity of radioembolization plus Sorafenib in advanced hepatocellular carcinoma: analysis of the European multicentre trial SORAMIC. Liver Int 2015;35:620-626.

14. 2018 Korean Liver Cancer Association-National Cancer Center Korea Practice Guidelines for the Management of Hepatocellular Carcinoma. Korean J Radiol 2019;20:1042-1113.

15. European Association for the Study of the Liver, Clinical practice guidelines panel; Wendon J, Panel M, Cordoba J, Dhawan A, et al. EASL Clinical Practical Guidelines on the management of acute (fulminant) liver failure. J Hepatol 2017;66:1047-1081.

16. European Association for the Study of the Liver. EASL Clinical Practice Guidelines: management of hepatocellular carcinoma. J Hepatol 2018;69:182-236.

17. Kudo M, Arizumi T. Transarterial chemoembolization in combination with a Molecular Targeted Agent: Lessons Learned from Negative Trials (Post-TACE, BRISK-TA, SPACE, ORIENTAL, and TACE-2). Oncology 2017;93 Suppl 1:127-134.

18. Forner A, Da Fonseca LG, Díaz-González Á, Sanduzzi-Zamparelli M, Reig M, Bruix J. Controversies in the management of hepatocellular carcinoma. JHEP Reps 2019;1:17-29.

19. Ricke J, Klümpen HJ, Amthauer $H$, Bargellini I, Bartenstein $P$, de Toni EN, et al. Impact of combined selective internal radiation therapy and sorafenib on survival in advanced hepatocellular carcinoma. J Hepatol 2019;71:1164-1174.

20. Chauhan N, Bukovcan J, Boucher E, Cosgrove D, Edeline J, Hamilton $\mathrm{B}$, et al. Intra-Arterial TheraSphere Yttrium-90 Glass Microspheres in the treatment of patients with unresectable hepatocellular carcinoma: protocol for the STOP-HCC Phase 3 randomized controlled trial. JMIR Res Protoc 2018;7:e11234. 